\title{
Citrus aurantium L. exhibits apoptotic effects on U937 human leukemia cells partly through inhibition of Akt
}

\author{
MIN HO HAN ${ }^{1}$, WON SUP LEE ${ }^{2}$, JING NAN LU ${ }^{2}$, GONSUP KIM $^{4}$, JIN MYUNG JUNG $^{3}$, \\ CHUNG HO RYU ${ }^{5}$, GI-YOUNG KIM ${ }^{6}$, HYE JIN HWANG $^{7}$, TAEG KYU KWON ${ }^{8}$ and YUNG HYUN CHOI ${ }^{1}$ \\ ${ }^{1}$ Department of Biochemistry, Dongeui University College of Oriental Medicine and Department of Biomaterial Control \\ (BK21 Program), Dongeui University Graduate School, Busan 614-052; Departments of ${ }^{2}$ Internal Medicine, and \\ ${ }^{3}$ Neurosurgery, Institute of Health Sciences, Gyeongsang National University School of Medicine, Jinju 660-702; \\ ${ }^{4}$ Institute of Life Science and School of Veterinary Medicine, ${ }^{5}$ Division of Applied Life Science (BK 21 Program) \\ Gyeongsang National University, Jinju 660-701; ${ }^{6}$ Laboratory of Immunobiology, Department of Marine Life Sciences, \\ Jeju National University, Jeju 690-756; ${ }^{7}$ Department of Food and Nutrition, Dongeui University, Busan 614-714; \\ ${ }^{8}$ Department of Immunology, School of Medicine, Keimyung University, Daegu 704-701, Republic of Korea
}

Received November 9, 2011; Accepted January 5, 2012

DOI: 10.3892/ijo.2012.1350

\begin{abstract}
Citrus fruits have been used as edible fruits and a traditional medicine since ancient times. In particular, the peels of immature citrus fruits are frequently prescribed in concert with other support herbs for many types of disease including cancer. We investigated the anti-proliferative activity of the peels of Citrus aurantium L. along with their effects on apoptosis. We prepared crude methanol extracts of the peels of Citrus aurantium L. (CMEs) and performed experiments using U937 human leukemia cells. The growth of U937 cells was inhibited by CME treatment in a dosedependent manner, and CME induced caspase-dependent apoptosis. CME inhibited the expression of XIAP and Bcl-xL which are anti-apoptotic proteins. CME inhibited Akt activity in a dose-dependent manner. The apoptotic activity of CME was significantly attenuated by Akt augmentation. In conclusion, this study suggested that CME should induce caspase-dependent apoptosis at least in part through Akt inhibition, providing evidence that CMEs have anticancer activity on human leukemia cells.
\end{abstract}

Correspondence to: Professor Won Sup Lee, Department of Internal Medicine, Institute of Health Sciences and Gyeongnam Regional Cancer Center, Gyeongsang National University School of Medicine, 90 Chilam-dong, Jinju 660-702, Republic of Korea

E-mail:1wshmo@hanmail.net or lwshmo@gshp.gsnu.ac.kr

Professor Yung Hyun Choi, Department of Biochemistry, Dongeui University College of Oriental Medicine, 42 San, Yangjung-dong, Busan 614-052, Republic of Korea

E-mail: choiyh@deu.ac.kr

Key words: Citrus aurantium L, Akt, apoptosis, leukemia, U937 cells

\section{Introduction}

Apoptosis is a process of programmed cell death that is crucial for maintaining the physiological balance of multicellular organisms. It has characteristic morphological changes, such as blebbing, cell shrinkage, nuclear fragmentation, chromatin condensation, and chromosomal DNA fragmentation $(1,2)$. It serves as a defense mechanism for cancer development by eliminating damaged cells which are prone to develop cancer (3). The process of apoptosis is controlled by a diverse range of cell signals, which may be triggered by either extrinsic or intrinsic inducers.

Flavonoids in fruits and vegetables are known to have or enhance anti-cancer activity by triggering apoptosis. Since studies suggest that high intake of fruit should reduce the risk of cancer and flavonoids in fruit can safely modulate the physiological function, much interest has been drawn to the possibility of controlling cancer with minimal toxicity (4-6).

Citrus aurantium L. which is called bitter orange is a flowering plant that belongs to the Rutaceae family of the order Sapindales, and is widely distributed in tropical and subtropical southeast regions of the world. The dried, entire immature peels of citrus fruit are employed in traditional herbal medicine and frequently prescribed in concert with other support herbs for many kind of disease including cancer. Case reports suggested that bitter orange supplements might be effective in treating angina (7), and ischemic colitis (8).

The flavoids isolated from citrus fruit including $C$. aurantium L. is mainly composed of hesperidin, naringenin, and nobiletin (9). These flavonoids have been reported to have some properties that regulate the inflammatory response and halt carcinogenesis (10). Recently some researchers have demonstrated that the crude methanol extract of Citrus aurantium L. $(\mathrm{CME})$ inhibits the nuclear factor- $\kappa \mathrm{B}(\mathrm{NF}-\kappa \mathrm{B})$ activity, and that the activation of $N F-\kappa B$ is involved in cancer cell survival and proliferation (11). Therefore, we hypothesized that CME has anticancer activity in cancer cells. Herein, we investigated the 
anti-proliferative activity of CME along with their effects on the apoptosis of U937 human leukemia cells.

\section{Materials and methods}

Cells and reagents. U937 human leukemia cells from the American type culture collection (Rockville, MD, USA) were cultured in RPMI-1640 medium (Invitrogen Corp., Carlsbad, CA, USA) supplemented with $10 \%$ (v/v) fetal bovine serum (FBS) (Gibco BRL, Grand Island, NY, USA), $1 \mathrm{mM}$ L-glutamine, $100 \mathrm{U} / \mathrm{ml}$ penicillin, and $100 \mu \mathrm{g} / \mathrm{ml}$ streptomycin at $37^{\circ} \mathrm{C}$ in a humidified atmosphere of $95 \%$ air and $5 \% \mathrm{CO}_{2}$. The CME was obtained from Professor G. Kim (Department of chemistry, Gyeongsang National University, Korea), and kept at $4^{\circ} \mathrm{C}$ with light shield. Antibodies against Bcl-2, Bax, Bad, Bcl-xL, XIAP, procaspase-3, -8, and -9, Fas, FasL, FADD, and ERK were purchased from Santa Cruz Biotechnology (Santa Cruz, CA, USA). poly(ADP-ribose) polymerase (PARP), and PLC $\gamma$-1 were purchased from PharMingen (San Diego, CA, USA). Peroxidase-labeled donkey anti-rabbit and sheep anti-mouse immunoglobulin, and an enhanced chemiluminescence (ECL) kit were purchased from Amersham (Arlington Heights, IL). Caspase activity assay kits were purchased from R\&D Systems (Minneapolis, MN, USA). All other chemicals not specifically cited here were purchased from Sigma Chemical Co. (St. Louis, MO). All these solutions were stored at $-20^{\circ} \mathrm{C}$. Stock solutions of DAPI $(100 \mu \mathrm{g} / \mathrm{ml})$ and propidium iodide (PI, $1 \mathrm{mg} / \mathrm{ml})$ were prepared in phosphate-buffered saline (PBS).

Cell viability assay. For the cell viability assay, the cells were seeded onto 24 -well plates at a concentration of $5 \times 10^{5}$ cells $/ \mathrm{ml}$, and then treated with the indicated concentration of CME for 24 and $48 \mathrm{~h}$. MTT $(0.5 \mathrm{mg} / \mathrm{ml})$ was subsequently added to each well. After $3 \mathrm{~h}$ of additional incubation, $100 \mu \mathrm{l}$ of a solution containing $10 \%$ SDS ( $\mathrm{pH} 4.8$ ) plus $0.01 \mathrm{~N} \mathrm{HCl}$ was added to dissolve the crystals. The absorption values at $570 \mathrm{~nm}$ were determined with an ELISA plate reader.

Nuclear staining with DAPI. After treatment with the indicated concentration of CME, the cells were harvested, washed with phosphate-buffered saline (PBS) and fixed with $3.7 \%$ paraformaldehyde in PBS for $10 \mathrm{~min}$ at room temperature. Fixed cells were washed with PBS and stained with $2.5 \mu \mathrm{g} / \mathrm{ml}$ 4,6-diamidino-2-phenylindole (DAPI) solution for $10 \mathrm{~min}$ at room temperature. The cells were washed two times with PBS, and analyzed under a fluorescent microscope.

Agarose gel electrophoresis for DNA fragmentation assay. The cells were lysed in a buffer containing $10 \mathrm{mM}$ Tris- $\mathrm{HCl}$ $\mathrm{pH} 7.4,150 \mathrm{mM} \mathrm{NaCl}, 5 \mathrm{mM}$ EDTA and $0.5 \%$ Triton X-100 on ice for $30 \mathrm{~min}$. The lysates were vortexed, then cleared by centrifugation at $10,000 \mathrm{x}$ g for $20 \mathrm{~min}$. The DNA in the supernatant was extracted using a 25:24:1 (v/v/v) equal volume of neutral phenol:chloroform:isoamyl alcohol and analyzed electrophoretically on $1.2 \%$ agarose gel containing $0.1 \mu \mathrm{g} / \mathrm{ml}$ ethidium bromide (EtBr).

Flow cytometry analysis for measurement of sub-G1 phase. After treatment of CME, the cells were collected, washed with cold PBS, and then centrifuged. The pellet was fixed in $75 \%$ (v/v) ethanol for $1 \mathrm{~h}$ at $4^{\circ} \mathrm{C}$. The cells were washed once with PBS and resuspended in cold PI solution $(50 \mu \mathrm{g} / \mathrm{ml})$ containing RNase A $(0.1 \mathrm{mg} / \mathrm{ml})$ in PBS (pH 7.4) for $30 \mathrm{~min}$ in the dark. Flow cytometry analyses were performed using FACSCalibur (Becton Dickinson, San Jose, CA). The sub-G1 population was calculated to estimate the apoptotic cell population (12).

Gel electrophoresis and western blot analysis. The cells were harvested and lysed, and protein concentrations were quantified using the BioRad protein assay (Bio-Rad Laboratories, Hercules, CA, USA). The proteins of the extracts were resolved by electrophoresis, electrotransferred to a polyvinylidene difluoride membrane (Millipore, Bedford, MA), and then the membrane was incubated with the primary antibodies followed by a conjugated secondary antibody to peroxidase. Blots were developed with an ECL detection system.

Assay of caspase-3, - 8 and -9 activity. The enzymatic activities of the caspases were determined by a colorimetric assay according to the manufacturer's protocol (R\&D Systems) in a kit for caspase activity. In brief, the cells were lysed in the supplied lysis buffer. The supernatants were collected and incubated with the supplied reaction buffer containing dithiothreitol and substrates at $37^{\circ} \mathrm{C}$. The reaction was measured by determining the change in absorbance at $405 \mathrm{~nm}$ using the microplate reader (13).

Statistics. Each experiment was performed in triplicate. The results were expressed as means \pm SD. Significant differences were determined using the one-way analysis of variance (ANOVA) with post-test Neuman-Keuls in the cases at least three treatment groups and Student's t-test for two group comparison. Statistical significance was defined as $\mathrm{P}<0.05$. Each experiment was performed at least in triplicate.

\section{Results}

CME inhibit proliferation and induced apoptosis in U937 human leukemia cells. To investigate the anti-tumor activity of CME, U937 cells were treated with various concentrations of CME for $24 \mathrm{~h}$. The growth of U937 cells were inhibited by $\mathrm{CME}$ treatment in a dose- and a time-dependent manner, and $\mathrm{IC}_{50}$ for $48 \mathrm{~h}-\mathrm{CME}$ treatment was $<40 \mu \mathrm{g} / \mathrm{ml}$ (Fig. 1A). To determine whether the decrease in viability of U937 cells caused by CME is related to apoptosis, we assessed the changes in nuclear morphology of CME-treated cells by DAPI staining. The staining revealed that cells with condensed and fragmented nuclei were observed in the cells treated by CME and the number of apoptotic cells increased in a concentration- and time-dependent manner (Fig. 1B). Next, we confirmed whether CME induced DNA fragmentation in U937 cells. As shown in Fig. 1C, CME induced a progressive accumulation of fragmented DNA, which appeared as a ladder pattern of DNA fragmentation at a concentration of $60 \mu \mathrm{g} / \mathrm{ml}$. Therefore, we measured cells with sub-G1 DNA content by flow cytometry to estimate the population of cell death. A significant accumulation of cells with sub-G1 DNA content was noted in a dose- and time-dependent manner (Fig. 1D). 
A

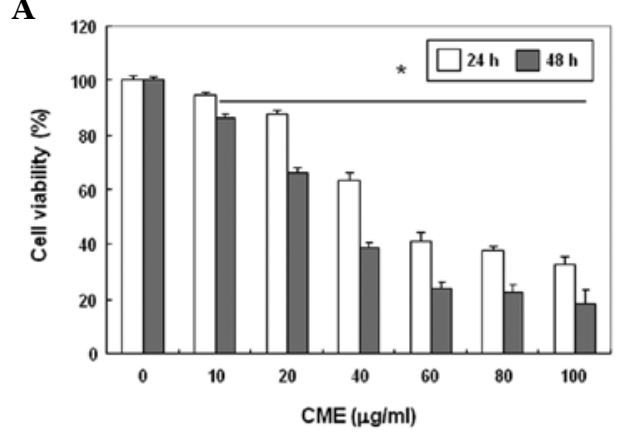

B

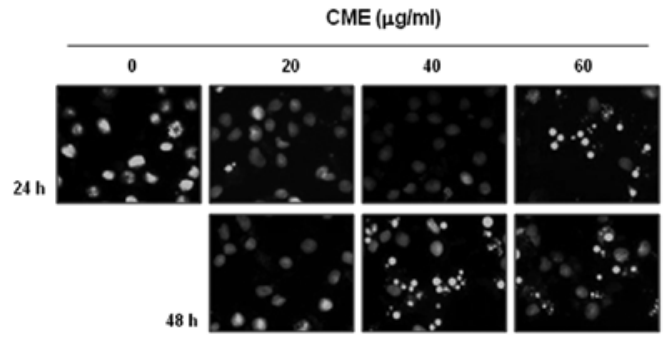

C
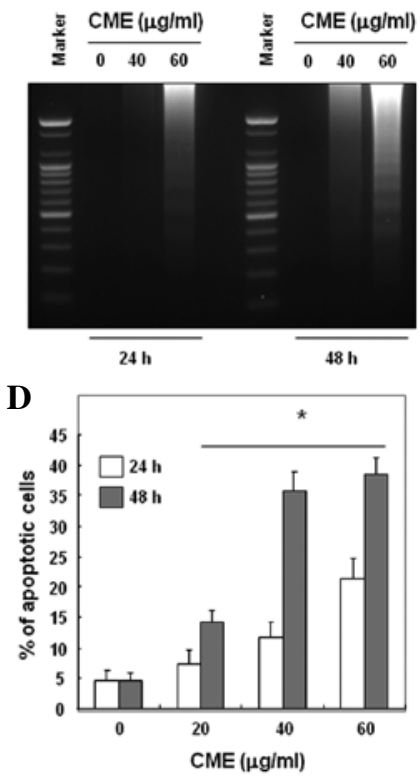

Figure 1. Growth inhibition and apoptosis induction by CME in U937 leukemia cells. The growth inhibition and cytotoxicity of CME are dose- and timedependent. U937 cells were seeded at the density of $5 \times 10^{4}$ cells per $\mathrm{ml}$. The cells were treated with indicated concentrations of CME for the indicated times. (A) Cell viability was analysized by MTT assay. The data are shown as means \pm SD of three independent experiments. "P $<0.05$ between the treated and the untreated control group. (B) After fixation, the cells were stained with DAPI solution. Stained nuclei were then observed under a fluorescent microscope using a blue filter (magnification, x400). (C) For the analysis of DNA fragmentation, genomic DNA was extracted and analyzed on $1.2 \%$ agarose gels. (D) To quantify the degree of CME-induced apoptosis, sub-G1 DNA content, which represents the fractions undergoing apoptotic DNA degradation, was analyzed by flow cytometry. The data are shown as means $\pm \mathrm{SD}$ of three independent experiments. " $\mathrm{P}<0.05$ between the treated and the untreated control group.

A

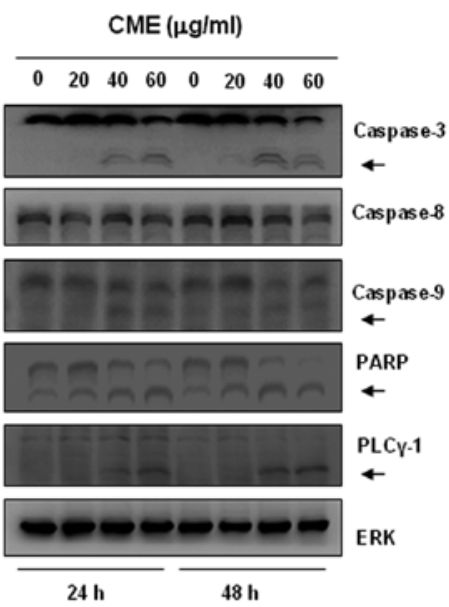

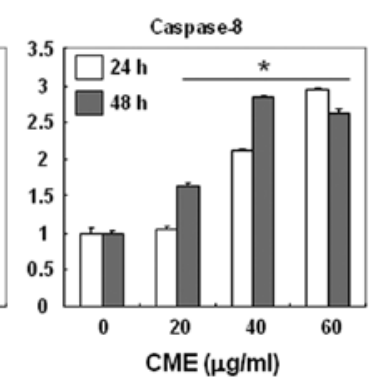

Figure 2. Activation of caspases and cleavage of PARP during CME-induced apoptosis in U937 cells. The activation of caspases and cleavage of PARP by CME are dose- and time-dependent. U937 cells were incubated at indicated concentrations of CME for the indicated times. (A) Total cell lysates were resolved by SDS-polyacrylamide gels and transferred onto nitrocellulose membranes. The membranes were probed with the anti-caspase- $3,-8,-9$ and anti-PARP antibodies. The proteins were visualized using an ECL detection system. ERK was used as an internal control. (B) The cell lysates from the cells treated with CME for the indicated times were assayed for in vitro caspase-3, - 8 and-9 activity using DEVD-pNA, IETD-pNA and LEHD-pNA, respectively, as substrates. The released fluorescent products were measured. Each bar graph represents mean \pm SD of three independent experiments. ${ }^{*} \mathrm{P}<0.05$ between the treated and the untreated control group.
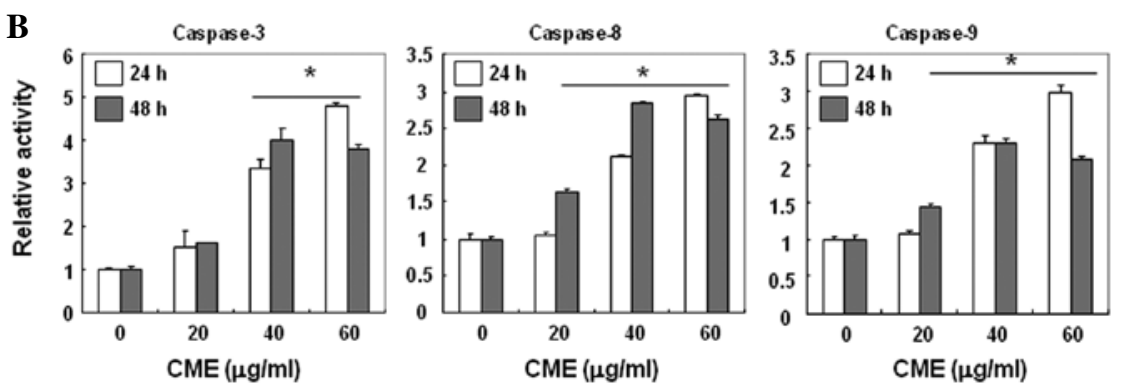

CME induces caspase activation and subsequent cleavage of PARP. Caspases are known as important mediators of apoptosis and contribute to leading cells undergoing apoptosis to irreversible cell death (3). By western blot analyses, we next assessed the expression of caspases and substrates (PARP and
PLC $\gamma$-1). CME decreased the expression levels of procaspase-3, -8 , and-9 in a dose- and time-dependent manner, which indicated caspase activation. The decrease in expression levels of procaspase- 3 is more prominent than that of procaspase- 8 in the cells treated with CME for $24 \mathrm{~h}$ (Fig. 2A). This finding 
A

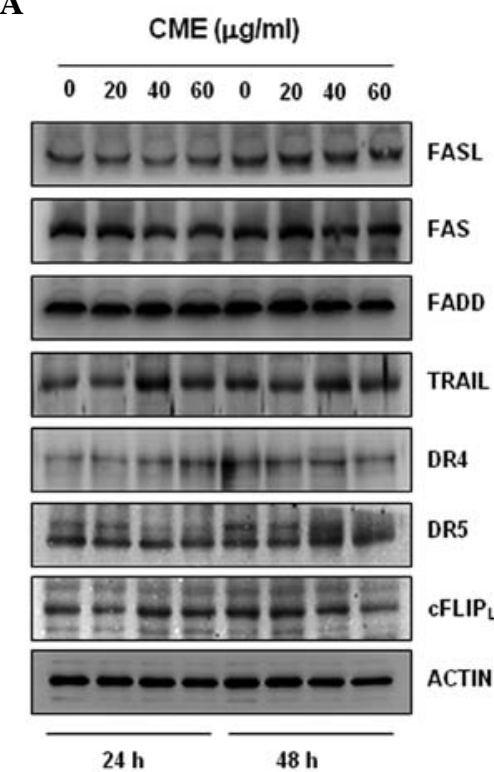

Figure 3. Role of death receptor pathway on activation of caspases in U937 leukemia cells. CME-induced apoptosis is not carried out through the death receptor-mediated pathway. The cells were incubated at indicated concentrations of CME for the indicated times. They were lysed and equal amounts of the proteins were resolved by SDS-polyacrylamide gels, and then transferred to nitrocellulose membranes. The membranes were probed with the indicated antibodies and detected by an ECL system. The results are from one representative of two independent experiments that showed similar patterns.

suggested that the activation of caspase- 8 should be related to the feedback pathway through caspase- 3 activation. Both the activation of caspases, and the cleavages of the substrates of caspase-3 (PARP and PLC $\gamma$-1) were observed to be dose- and a time-dependent (Fig. 2A). These findings suggest that CME induces apoptosis through caspase activation. To confirm and quantify the proteolytic activation of caspases, we assessed their activities. Caspase activity assay confirmed that CME increased proteolytic activities of caspases in a dose- and a time-dependent manner (Fig. 2B).

CME-induced apoptosis is not carried out through the death receptor-mediated pathway. To determine which apoptotic pathway is involved in the CME-induced apoptosis, we measured the expression of death receptors (DR4, DR5, and Fas-receptor) and corresponding pro-apoptotic ligands [TNF-related apoptosis-inducing ligand (TRAIL), and Fas ligand] as well as an adaptor molecule, Fas-associated protein with death domain (FADD). We found no differences in the expression levels before and after the CME treatment (Fig. 3), suggesting that CME may induce apoptosis through the intrinsic pathway and that the activation of caspase- 8 may be related to the feedback pathway through caspase-3 activation.

CME induces apoptosis by modulating Bcl-2 and IAP family members. BID is a pro-apoptotic $\mathrm{Bcl}-2$ protein containing only the BH3 domain (14). In response to apoptotic signaling, BID interacts with another Bcl-2 family protein, Bax, leading to the insertion of Bax into the mitochondrial outer membrane and induce the opening of the mitochondrial voltage-dependent anion channel (VDAC). This results in the release of cytochrome $\mathrm{c}$ and other pro-apoptotic factors from the mitochondria,
A

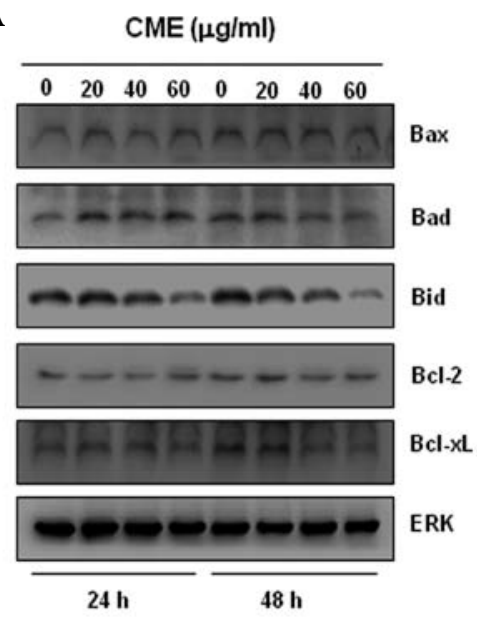

B

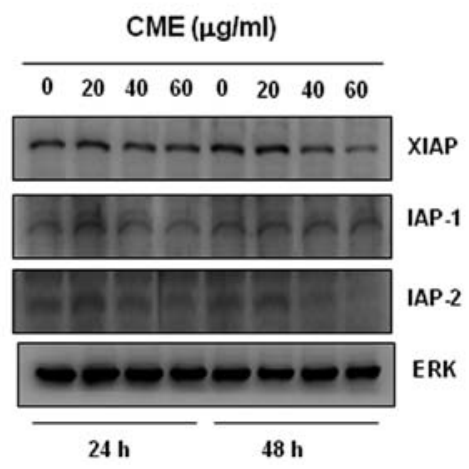

Figure 4. Regulation of Bcl-2 and IAP family members during CME-induced apoptosis. CME suppresses the expression of Bcl-2, Bcl-xL, XIAP, and IAP-2 in a dose- and time-dependent manner. The cells were incubated at the indicated concentrations of CME for the indicated times, and lysed. Equal amounts of the cell lysate were separated by SDS-polyacrylamide gels and then transferred to nitrocellulose membranes. The membranes were probed with the indicated antibodies and detected by an ECL detection system. To confirm equal loading, the blot was stripped of the bound antibody and reprobed with the anti-ERK antibody. (A) Bcl-2 family members. (B) IAP family members. The results are from one representative experiment of at least three independent experiments that showed similar patterns.

leading to activation of caspases. To confirm that CME induced apoptosis and to elucidate further underlying mechanisms of CME-induced apoptosis, we assessed the levels of Bcl-2 family members, which play a crucial role in apoptosis. Western blotting revealed CME induced BID activation in a dose- and a time-dependent manner whereas the expression of Bcl-2 and Bcl-xL remained unchanged or slightly reduced (Fig. 4A). While the Bax expression remained unchanged during CME treatment, the Bax/Bcl-2 ratio was increased (Fig. 4B). These finding suggested that reduction in $\mathrm{Bcl}-2$ expression leading to an increase in Bax/Bcl-2 ratio should be an important mechanism of CME-induced apoptosis in U937 cells. The expression of Bcl-2-associated death promoter (BAD) protein was also increased in U937 cells treated by CME for $24 \mathrm{~h}$. These findings suggested that $\mathrm{CME}$ induced apoptosis by modulating $\mathrm{Bcl}-2$ family members, especially the Bax/Bcl-2 ratio as well as BID.

The inhibitors of apoptosis (IAP) serve as endogenous inhibitors of apoptosis (15). The human IAP family consists of 8 members, and the best characterized IAP is the X-linked inhibitor of apoptosis protein (XIAP), which binds caspase-9, -3 and -7 , thereby inhibiting their activation and preventing 
A
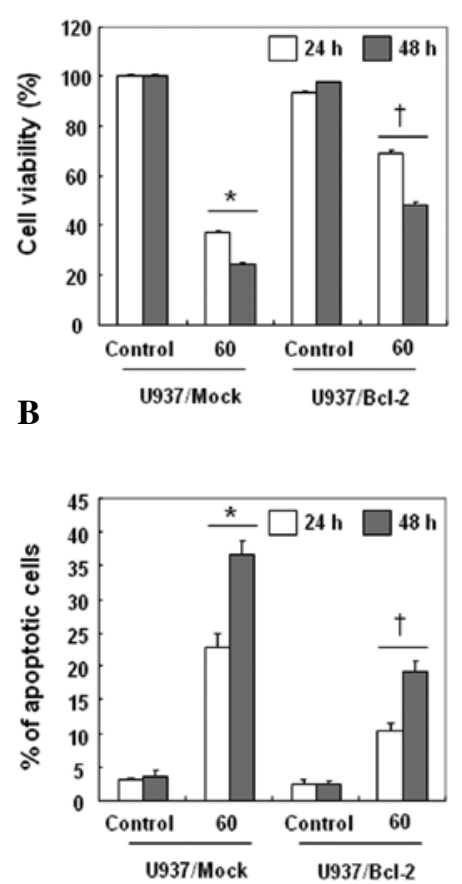

C

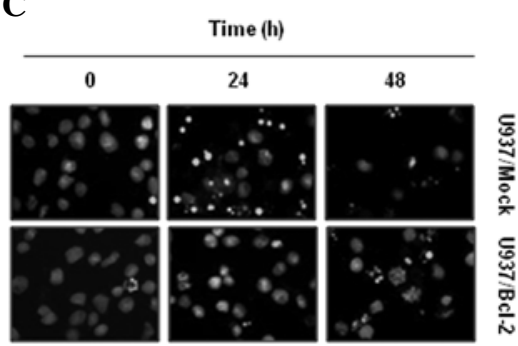

D

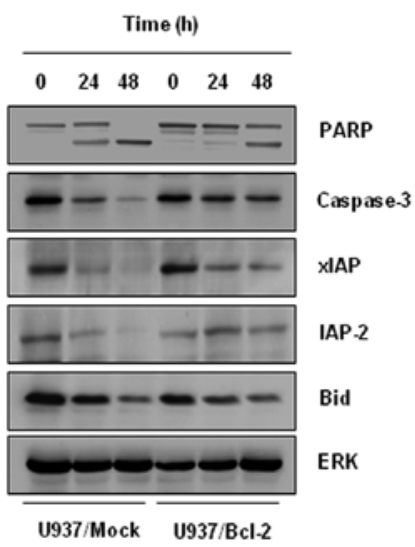

Figure 5. Effects of Bcl-2 overexpression in U937 cells on CME-induced apoptosis. Overexpression of Bcl-2 suppresses the induction of CME-induced caspase-3 activation and PARP cleavages. (A) U937/ vector or U937/Bcl-2 cells were treated with $60 \mu \mathrm{g} / \mathrm{ml}$ of CME for the indicated times. Cell viability was assessed by MTT assay. (B) To quantify the degree of CME-induced apoptosis, sub-G1 DNA content was analyzed by flow cytometry. The data are shown as means \pm SD of three independent experiments. The data are shown as means \pm SD of three independent experiments. ${ }^{*} \mathrm{P}<0.05$ between the groups treated with and without CME, ${ }^{+} \mathrm{P}<0.05$ between the U937/vector and U937/Bcl-2 cells. (C) After fixation, the cells were stained with DAPI solution. Stained nuclei were then observed under fluorescent microscope using a blue filter (magnification, x400). (D) The cells were lysed, equal amounts of the lysate were separated by SDS-polyacrylamide gels, and then transferred to nitrocellulose membranes. The membranes were probed with the indicated antibodies and detected by an ECL detection system. To confirm equal loading, the blot was stripped of the bound antibody and reprobed with the anti-ERK antibody.

apoptosis. cIAP1 and cIAP2 have also been shown to bind caspases although the IAP inhibiting process is not fully understood. Herein we also assessed the levels of IAP family members (XIAP, cIAP1 and cIAP2). Western blotting revealed CME suppressed the expression of XIAP, cIAP1 and cIAP2 in a dose- and a time-dependent manner in U937 cells (Fig. 4C). These finding suggested that CME also augmented apoptosis by suppressing IAP family members in U937 cells.

Bcl-2 overexpression suppresses CME-induced apoptosis. $\mathrm{Bcl}-2$ is an anti-apoptotic protein which is thought to be involved in resistance to conventional cancer treatment. The reduced expression of Bcl-2 family members and XIAP family members observed in CME-treated cells may result from degradation of proteins due to apoptotic process. Hence, we evaluated the effects of Bcl-2 overexpression on Bcl-2 and IAP family members suppressed by CME as well as on the CME-induced apoptosis by comparing those between U937/ vector and U937/ $\mathrm{Bcl}-2$ cells that constitutively express high levels of Bcl-2. As shown in Fig. 5A and B, Bcl-2 overexpression significantly suppressed apoptosis as well as the reduction in cell viability in response to $\mathrm{CME}$ treatment. We also assessed the changes in nuclear morphology of CME-treated cells by DAPI staining. The staining showed that $\mathrm{Bcl}-2$ overexpression reduced the development of condensed and fragmented nuclei in the CME-treated U937 cells (Fig. 5C). To confirm this finding at the molecular level, we performed western blotting for the molecules involved in CME-induced apoptosis (caspase-3, PARP, BID, Bad, XIAP, IAP2). The western blotting revealed that $\mathrm{Bcl}-2$ overexpression suppressed the activation of BID and caspase-3, and the subsequent cleavages of PARP, but CME-induced BID activation and XIAP suppression were not greatly affected (Fig. 5D). These finding suggested that CME-induced apoptosis is closely related to BID and XIAP activation.

CME induces apoptosis by suppressing Akt phosphorylation in U937 cells. PI3K/Akt is important in regulating cell proliferation and apoptosis. The expression of pro-apoptotic Bad and anti-apoptotic XIAP proteins is regulated by Akt. Therefore, we investigated the effects of CME on Akt in U937 cells. Since the activity of Akt is regulated by phosphorylation, we assessed the levels of phosphorylated Akt in U937 cells treated with CME. By western blotting revealed that CME suppressed the phosphorylation of Akt in a dose- and a time-dependent manner (Fig. 6A). To confirm this finding, we evaluated the effects of CME on U937/Akt cells that express constitutively active Akt in terms of apoptosis by comparing those between U937/vector and U937/Akt cells. As shown in Fig. 6B and C, Akt activation led to significant suppression of apoptosis as well as the reduction in cell viability in response to CME treatment. We also assessed the changes in nuclear morphology of CME-treated cells by DAPI staining. The DAPI staining showed that overexpression of activated Akt reduced the occurrence of condensed and 
A

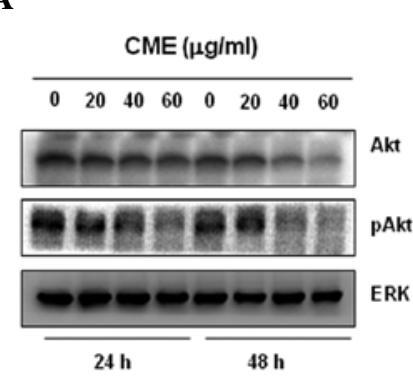

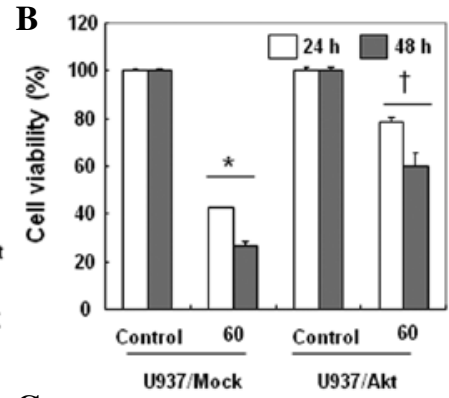

C

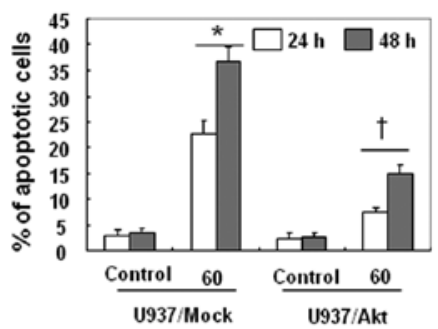

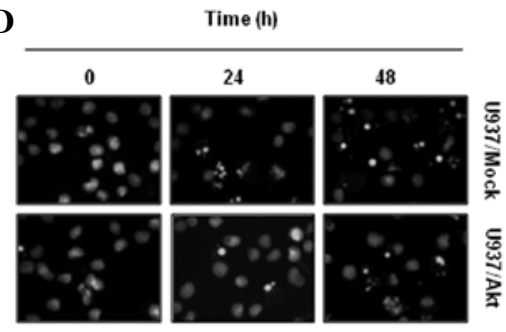

$\mathbf{E}$

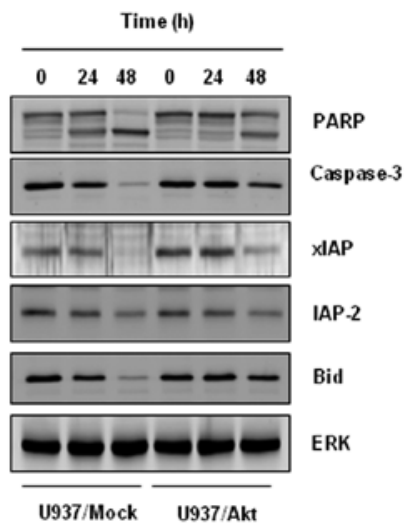

Figure 6. Effects on protein kinase B (PKB/Akt) in U937 cells on CME-induced apoptosis. Overexpression of Akt augments XIAP and suppresses the induction of CME-induced caspase-3 activation and PARP cleavages. (A) U937/vector or U937/Akt cells were treated with $60 \mu \mathrm{g} / \mathrm{ml}$ of CME for the indicated times. Cell viability was assessed by MTT assay. (B) To quantify the degree of CME-induced apoptosis, sub-G1 DNA content was analyzed by flow cytometry. The data are shown as means \pm SD of three independent experiments. ${ }^{\circ}<0.05$ between the groups treated with and without $\mathrm{CME}$, ${ }^{\dagger} \mathrm{P}<0.05$ between the $\mathrm{U} 937 / \mathrm{vector}$ and U937/Akt cells. (C) After fixation, the cells were stained with DAPI solution. Stained nuclei were then observed under fluorescent microscope using a blue filter (magnification, x400). (D) The cells were lysed, equal amounts of the lysate were separated by SDS-polyacrylamide gels, and then transferred to nitrocellulose membranes. The membranes were probed with the indicated antibodies and detected by an ECL detection system. To confirm equal loading, the blot was stripped of the bound antibody and reprobed with the anti-ERK antibody.

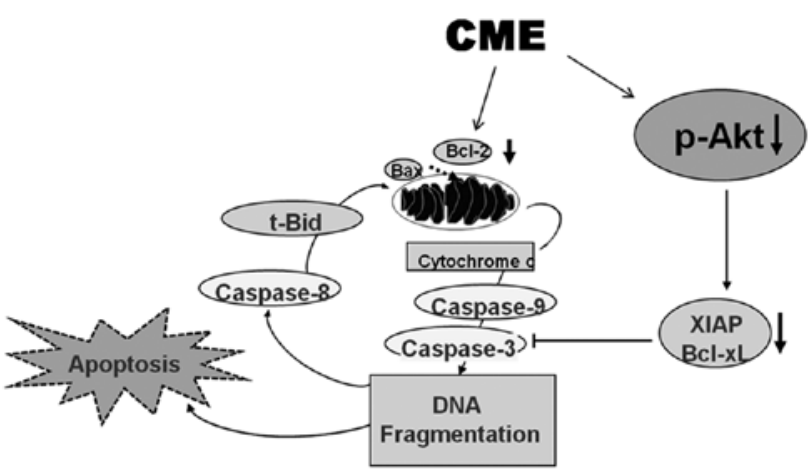

Figure 7. Schematic representation on apoptotic effects of CME on U937 human leukemia cells. CME suppresses expression of XIAP through inhibition of p-Akt, this inhibition causes sequential activation of caspase-3 resulting in apoptosis. In addition, $\mathrm{CME}$ reduced Bcl-2 expression an apoptotic protein and then augmented apoptosis. Taken together, this study suggested that Akt is an important protein kinase that regulates the apoptotic effect of CME and the suppression of Bcl-2 is an important factor in inducting apoptosis in human leukemia U937 cells.

fragmented nuclei in the CME-treated U937 cells (Fig. 6D). To confirm this finding at the molecular level, we performed western blotting for the molecules involved in CME-induced apoptosis (caspase-3, PARP, BID, Bad, XIAP, IAP2). Western blotting revealed that Akt activation suppressed the activation of BID and caspase-3, and the subsequent cleavages of PARP (Fig. 6E). These findings suggested that CME should induce apoptosis at least in part through activation of Akt.

\section{Discussion}

This study was designed to determine whether CME has anti-cancer properties in human leukemia cells, specifically of apoptosis and to further investigate the underlying mechanisms of the anticancer effects of CME. Herein, we found that CME induced caspase-dependent apoptosis in a dose-dependent manner. The induction of apoptosis was triggered through the intrinsic pathway by modulating Bcl-2 and IAP family members related to activation of Akt. Evidence suggested that apoptosis, a type of programmed cell death is an underlying mechanism by which various anti-cancer and chemopreventive agents including natural compounds exert anti-cancer effects (4-6). Apoptosis is triggered by the activation of a set of death effector cysteine proteases called caspases and their activation plays an important role in apoptosis. In most of the apoptotic processes, caspase- 3 has been shown to play a pivotal role in the terminal and execution phase of apoptosis $(16,17)$. This study demonstrated that CME induced caspase-3 activation and the subsequent cleavages of PARP ( $89 \mathrm{kDa})$. The cleavage of PARP ( $89 \mathrm{kDa}$ ) indicates early apoptosis (18). This finding is consistent with previous finding that hesperidin can induce caspase-3-dependent apoptosis in cancer cells (19). Apoptosis is an endogenous programmed cell death which can be triggered through either the intrinsic pathway or the extrinsic pathway. We excluded the possibility that the CME-induced apoptosis in U937 cells is related to extrinsic apoptosis by checking the expression of death receptors and their ligands. 
Bcl-2 and IAP family members are very important in regulating apoptosis $(3,15)$. We also found that CME suppressed anti-apoptotic proteins XIAP and IAP-2, activated the proapoptotic proteins BID and Bad, and increased the Bax/ $\mathrm{Bcl}-2$ ratio. Furthermore, this study demonstrated that the Bcl-2 overexpression suppressed caspase-3 activation and subsequent apoptosis, but CME-induced BID activation and XIAP suppression was not greatly influenced. These findings suggest that CME-induced apoptosis is closely related to BID and XIAP activation. BID is a pro-apoptotic protein containing only the BH3 domain. BID interacts with Bax, leading to the insertion of Bax into the outer mitochondrial membrane in response to apoptotic signaling (3). Therefore, Bax expression in whole cell lysates was not induced by CME in this study, but $\mathrm{Bax}$ in the mitochondrial fraction increases and induces mitochondrial dysfunction which is the key step in triggering apoptosis through the intrinsic pathway.

Akt plays a critical role in regulating cell proliferation and apoptosis, and it is an upstream signal controlling Bcl-2 and IAP family members such as pro-apoptotic Bad and antiapoptotic XIAP proteins (20). The Akt activity was regulated by phosphorylation. In this study, we clearly demonstrated that CME inhibited Akt phosphorylation and the suppression of Akt phosphorylation was closely related to apoptosis.

In summary, this study demonstrated that CME suppressed the cell viability and induced caspase-dependent apoptosis in U937 cells. The induction of apoptosis was triggered through intrinsic pathway by modulating $\mathrm{Bcl}-2$ and IAP family members. In addition, suppression of Akt phosphorylation was involved in CME-induced apoptosis in U937 cells (Fig. 7). This study provides evidence that CME might have anticancer properties in leukemia cells.

\section{Acknowledgements}

This study was supported by grants from the National R\&D Program for Cancer Control, Ministry of Health \& Welfare, Republic of Korea (0820050).

\section{References}

1. Buendia B, Santa-Maria A and Courvalin JC: Caspase-dependent proteolysis of integral and peripheral proteins of nuclear membranes and nuclear pore complex proteins during apoptosis. J Cell Sci 112: 1743-1753, 1999.

2. Kerr JF, Wyllie AH and Currie AR: Apoptosis: A basic biological phenomenon with wide-ranging implications in tissue kinetics. Br J Cancer 26: 239-257, 1972.
3. Hengartner MO: The biochemistry of apoptosis. Nature 407: 770-776, 2000

4. de Sousa RR, Queiroz KC, Souza AC, Gurgueira SA, Augusto AC Miranda MA, Peppelenbosch MP, Ferreira CV and Aoyama H: Phosphoprotein levels, MAPK activities and NFkappaB expression are affected by fisetin. J Enzyme Inhib Med Chem 22: 439-444, 2007.

5. Liu BL, Zhang X, Zhang W and Zhen HN: New enlightenment of French paradox: Resveratrol's potential for cancer chemoprevention and anti-cancer therapy. Cancer Biol Ther 6: 1833-1836, 2007.

6. Chun KH, Kosmeder JW II, Sun S, Pezzuto JM, Lotan R, Hong WK and Lee HY: Effects of deguelin on the phosphatidylinositol 3-kinase/Akt pathway and apoptosis in premalignant human bronchial epithelial cells. J Natl Cancer Inst 95: 291-302, 2003.

7. Gange CA, Madias C, Felix-Getzik EM, Weintraub AR and Estes NA III: Variant angina associated with bitter orange in a dietary supplement. Mayo Clin Proc 81: 545-548, 2006.

8. Sultan S, Spector J and Mitchell RM: Ischemic colitis associated with use of a bitter orange-containing dietary weight-loss supplement. Mayo Clin Proc 81: 1630-1631, 2006.

9. Nogata Y, Sakamoto K, Shiratsuchi H, Ishii T, Yano M and Ohta H: Flavonoid composition of fruit tissues of citrus species. Biosci Biotechnol Biochem 70: 178-192, 2006.

10. Manthey JA, Grohmann K and Guthrie N: Biological properties of citrus flavonoids pertaining to cancer and inflammation. Curr Med Chem 8: 135-153, 2001.

11. Aggarwal BB: Nuclear factor-kappaB: The enemy within. Cancer Cell 6: 203-208, 2004

12. Cui ZG, Hong NY, Guan J, Kang HK, Lee DH, Lee YK and Park DB: cAMP antagonizes ERK-dependent antiapoptotic action of insulin. BMB Rep 44: 205-210, 2011.

13. Cho SY, Lee JH, Bae HD, Jeong EM, Jang GY, Kim CW, Shin DM, Jeon JH and Kim IG: Transglutaminase 2 inhibits apoptosis induced by calcium-overload through down-regulation of Bax. Exp Mol Med 42: 639-650, 2010.

14. Yu F, Watts RN,Zhang XD, Borrow JM and Hersey P: Involvement of BH3-only proapoptotic proteins in mitochondrial-dependent Phenoxodiol-induced apoptosis of human melanoma cells. Anticancer Drugs 17: 1151-1161, 2006.

15. Deveraux QL and Reed JC: IAP family proteins - suppressors of apoptosis. Genes Dev 13: 239-252, 1999.

16. Ashkenazi A: Targeting death and decoy receptors of the tumournecrosis factor superfamily. Nat Rev Cancer 2: 420-430, 2002.

17. Thornberry NA and Lazebnik Y: Caspases: enemies within. Science 281: 1312-1316, 1998.

18. Tewari M, Quan LT, O'Rourke K, Desnoyers S, Zeng Z, Beidler DR, Poirier GG, Salvesen GS and Dixit VM: Yama/CPP32 beta, a mammalian homolog of CED-3, is a CrmA-inhibitable protease that cleaves the death substrate poly(ADP-ribose) polymerase. Cell 81: 801-809, 1995.

19. Park HJ, Kim MJ, Ha E and Chung JH: Apoptotic effect of hesperidin through caspase 3 activation in human colon cancer cells, SNU-C4. Phytomedicine 15: 147-151, 2008.

20. Franke TF, Hornik CP, Segev L, Shostak GA and Sugimoto C: PI3K/Akt and apoptosis: size matters. Oncogene 22: 8983-8998, 2003. 